\title{
Characterization of liquefied waste bamboo and white-rotted wood
}

\author{
Q. Wang ${ }^{1}$, H. Takahashi ${ }^{1}$, Y. Takahashi ${ }^{1}$, H. Kurokawa ${ }^{1}$, \\ K. Sekiguchi ${ }^{1} \&$ K. Sugiyama ${ }^{2}$ \\ ${ }^{I}$ Graduate School of Science and Engineering Saitama University, Japan \\ ${ }^{2}$ Hachinohe National College of Technology, Japan
}

\begin{abstract}
Due to the depletion of fossil fuel resources, the utilization of sustainable and renewable biomass is studied in various fields. Wood liquefaction is one of the applicable methods for material utilization of waste biomass. In this study, valueless waste bamboo and white-rotted wood were liquefied for reuse as an alternative plastic material. The waste bamboo samples were liquefied in polyethylene glycol (PEG) with sulfuric acid as a catalyst. Then, the molecular weight distribution of the contents of the liquefied bamboo samples was analyzed using gel permeation chromatography (GPC) and the residue mass was also measured in order to investigate the residue contents. At the same time, the whiterotted woody samples were liquefied in phenol with sulfuric acid as a catalyst. Rotted woody samples and liquefied contents were analyzed with X-ray diffraction (XRD) and determined by GPC, and the residue contents were also compared with the results of non-rotted woody samples.

The waste bamboo samples were liquefied effectively under the reaction conditions of a temperature of $150^{\circ} \mathrm{C}$, the acidic catalyst of $12 \% \mathrm{H}_{2} \mathrm{SO}_{4}$ and $1 / 4$ proportion of bamboo samples to solvent, respectively. The residue contents were the lowest at a reaction time of 30 minutes, and after that they were increased a reaction progressed. With the help of GPC analysis, it was found that the peaks of the contents of the liquefied bamboo samples at the initial reaction stage appeared at a high molecular weight distribution, which disappeared when the reaction progressed. When the white-rotted woody samples were liquefied, due to the low powder density, the samples did not perform well at the initial reaction stage. From GPC analysis of the liquefied contents, the liquefied tendency and the residue
\end{abstract}


contents of the white-rotted woody samples seemed to be almost the same as those of the non-rotted original woody samples.

Keywords: liquefaction, waste bamboo, waste wood, white-rot fungi.

\section{Introduction}

In recent years, it has become problematic to use fossil fuel resources because of their depletion in consumeristic society and harm to the global environment. Therefore, new resources, as an alternative to fossil fuel, are being studied. Biomass is one of the new resources which are reusable and sustainable. In Japan, the habitat of bamboo - one of the biomass - has increased in contrast to a decrease in demand of material utilization [1]. Moreover, thinning woods are usually left as waste woody materials in their forest. The waste woody materials often decay because of exposure to rain and the absence of preservative treatment. Using bamboo and rotten wood in this condition contribute to promoting more effective utilization of unused and lower cost biomass. Wood liquefaction is one of the effective technologies for biomass utilization [2]. When a woody powder is mixed with an organic solvent in the presence of an acid catalyst and heated, a black viscous liquid material is formed. This material is called liquefied wood and can form resin by adding chemical reagents $[3,4]$. Therefore, wood liquefaction has the advantage of utilizing unused biomass and saving fossil fuel resources because such kind of resin can substitute the plastic materials made from fossil fuel resources.

In this paper, the liquefaction behavior and efficient reaction condition of the waste bamboo samples were investigated. At the same time, the rotted woody samples were liquefied, and the liquefaction behavior was also compared with that of the non-rotted woody samples. Among two kinds of fungi classified by the woody rot styles of brown-rot fungi and white-rot fungi, brown-rotted wood liquefies better than non-rotted wood [5]. From this point of view, it is suggested that white-rotted wood may also liquefy better than non-rotted wood. Because there are no reports of liquefaction of white-rotted wood, white-rotted woody samples were therefore also applied in this study.

\section{Materials and methods}

\subsection{Preparation of bamboo and rotted woody powder samples}

The bamboo samples were obtained from a Chichibu wood-processing firm of the Saitama prefecture, Japan. The bamboo samples were crushed and classified from $0.5-1.0 \mathrm{~mm}$ through a sieve, dried for 24 hours in an oven at $105^{\circ} \mathrm{C}$ and used as bamboo powder samples, so-called "bamboo samples".

While using the white-rotted woody samples, Japanese cherry samples were also collected from another Chichibu wood-processing firm of the Saitama prefecture, which had white-rotted sapwood and non-rotted heartwood and separated sapwood from heartwood. The separated sapwood and heartwood were experimented on as rotted woody samples and non-rotted woody samples 
respectively. All the woody samples were classified to $<250 \mu \mathrm{m}$ and $250-500 \mu \mathrm{m}$ through a sieve, dried for 24 hours in an oven at $105^{\circ} \mathrm{C}$ and used as woody powder samples, namely "woody samples".

\subsection{Liquefaction of bamboo samples and analysis of liquefied material}

\subsubsection{Liquefaction experiment of bamboo samples}

The bamboo samples, a mixed solution (1/9 proportion of glycerin to polyethylene glycol) and the catalyst $\left(\mathrm{H}_{2} \mathrm{SO}_{4}\right)$ were measured as they were needed, respectively and then mixed in a $300 \mathrm{~mL}$ three-neck reaction flask equipped with a stirrer and a reflux condenser [6]. Then, the reaction flask was immersed in a preheated oil bath. After different liquefaction reaction times of 10, 30, 90, 180 and 300 minutes, the liquefied woody samples in the flask were collected and put into a vial in order to measure the residue contents and analyze. And then the vial was immersed in cold water to stop the liquefaction reaction. To filter the residue, $10 \mathrm{~mL}$ of methanol was added to the vial and combined completely using a shaking apparatus. Then the filtered residue was dried for 24 hours in an oven at $105^{\circ} \mathrm{C}$. The rate of residue was calculated as shown in the following equation (1).

$$
\text { Ratio of residue }(\%)=R / R_{i} \times 100
$$

$R=$ the weight ratio $(\%)$ of residue in the sampling vial.

$R_{i}=$ the initial weight ratio (\%) of woody powder samples in the reaction flask.

The frequently-used index to investigate the optimal condition for liquefaction is the rate of residue. Residue is the unreacted woody samples or the unresinified material generated when a condensation reaction occurred in the liquefied woody samples. In general, the lower residue contents had to be examined. Therefore, we had to change liquefaction reaction temperature, amount of catalyst and solvent, and then investigate the amount of residue.

\subsubsection{Measurement of molecular weight distribution of liquefied bamboo samples}

The molecular weight distribution of the contents of liquefied bamboo samples was analyzed using gel permeation chromatography (GPC) equipped with a column (KF-802, Shodex, Japan), HPLC pump (PU-2080, Jasco, Japan), column thermostat (CO-2060, Jasco, Japan) and RI detector (RI-2031, Jasco, Japan). $10 \mathrm{mg}$ of the liquefied bamboo samples were diluted with $10 \mathrm{~mL}$ of tetrahydrofuran (THF), then, the mixed solution was analyzed starting with GPC. The analytical conditions are shown in Table 1.

\subsection{Analysis and liquefaction of white-rotted woody samples and measurement of molecular weight distribution of liquefied contents}

\subsubsection{Holocellulose and lignin measuring in white-rotted wood samples}

To analyze the components of wood, woody samples were defatted by soxhlet extraction. Using these defatted woody samples, the contents of holocellulose and lignin in the wood were analyzed by the Wise method and the Klason lignin 
method respectively. The detail experiments were performed according to the procedure given in "Mokusitu Kagaku Jikken Manual" [7].

Table 1: Analytical conditions of GPC analysis.

\begin{tabular}{ll} 
Mobile phase & THF \\
Flow rate & $1 \mathrm{~mL} / \mathrm{min}$ \\
Dilute concentration of sample & $0.1 \mathrm{w} / \mathrm{v} \%$ in THF \\
Column temp. & $40^{\circ} \mathrm{C}$ \\
Volume of sample loop & $100 \mathrm{~L}$ \\
\hline
\end{tabular}

\subsubsection{Measurement of crystallinity of white-rotted woody samples}

Using X-ray diffraction (Rint UltimaIII, Rigaku Corporation, Japan), the crystallinity of holocellulose of woody samples was measured. By measuring crystallinity and comparing rotted woody samples with non-rotted woody samples, it was confirmed that the wood had decayed. The analysis condition was determined by X-ray $40 \mathrm{kV} / 40 \mathrm{~mA}$, a $2 / 3^{\circ}$ emission slit, $2 / 3^{\circ}$ dispersion slit, a light receiving slit of $0.3 \mathrm{~mm}$, and a monochromatic light receiving slit of $0.8 \mathrm{~mm}$.

\subsubsection{Liquefaction of white-rotted woody samples and measurement of molecular weight distribution of white-rotted woody samples}

The woody samples, phenol and catalyst $\left(\mathrm{H}_{2} \mathrm{SO}_{4}\right)$ were each measured when necessary and then mixed in a $300 \mathrm{~mL}$ three-neck reaction flask equipped with a stirrer and a reflux condenser [8]. Then, the reaction flask was immersed in a preheated oil bath. After various periods of 15, 30, 60, 90 and 120 minutes, the liquefied woody samples in the flask were collected and put into a vial in order to measure the residue contents and analyze. And then, the vial was immersed in cold water to stop the liquefaction reaction. To filter the residue, $10 \mathrm{~mL}$ of methanol was added to the vial and combined completely using a shaking apparatus. Then the filtered residue was dried for 24 hours in an oven at $105^{\circ} \mathrm{C}$. The rate of residue was calculated (as shown in equation (1)). The measurement of molecular weight distribution was performed using the method mentioned in Section 2.2.2.

\section{Results and discussions}

\subsection{Result of the liquefaction experiment of bamboo samples and measurement of molecular weight distribution of liquefied contents}

\subsubsection{Optimal liquefaction condition of bamboo samples: the influence of reaction temperature}

Liquefaction reaction conditions were arranged for an amount of catalyst $\left(\mathrm{H}_{2} \mathrm{SO}_{4}\right)$ of $12 \%$ (for the amount of bamboo samples) and 1/4 proportion of bamboo samples to solvent, separately, and liquefaction reaction temperatures were changed to 120,150 and $180^{\circ} \mathrm{C}$. As shown in Figure 1, the initial rate of reaction increased as the reaction temperature increased. After that, the increments in the rates of residue were observed due to the condensation reaction. Although 
the beginning rate of residue at $120^{\circ} \mathrm{C}$ was higher than those of other high temperatures, the condensation reaction at a later reaction stage after 100 minutes did not occur. Conversely, the rate of residue at $180^{\circ} \mathrm{C}$ was minimized at a reaction time of 10 minutes; different from other low temperatures; the condensation reaction at the following reaction stage after 30 minutes was noticeable. Because the liquefaction reaction progressed extremely at a much higher rate under the reaction temperature of $180^{\circ} \mathrm{C}$, all polyol in the reactant solution seemed to be almost consumed. As a result, the condensation reaction occurred as a result of the vacant reaction sites being combined. Therefore, it shows that the optimum reaction temperature is $150^{\circ} \mathrm{C}$.

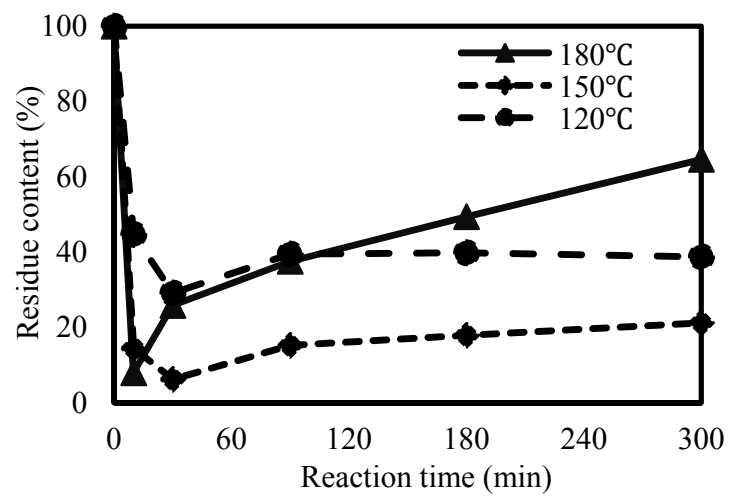

Figure 1: The effect of the reaction temperatures on residue contents.

\subsubsection{Optimal liquefaction condition of bamboo samples: influence of amount of catalyst}

Liquefaction reaction conditions were arranged for a reaction temperature of $150^{\circ} \mathrm{C}$ and $1 / 4$ proportion of bamboo samples to solvent and the catalyst amounts were changed into 12, 24 and $48 \%$ (as shown in Figure 2). If the catalyst quantity

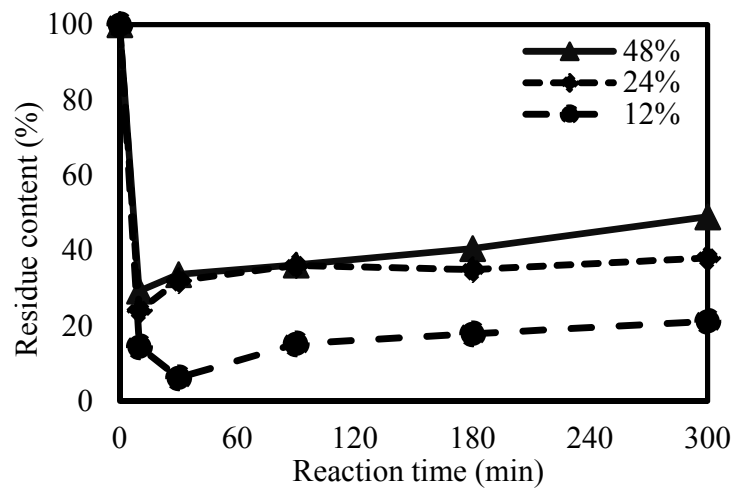

Figure 2: The effect of the catalyst amounts on residue contents. 
increased, a reaction seemed to improve. However, the rate of residue was the lowest at $12 \%$ of catalyst. It is thought that the condensation reaction competed dominantly with the degradation reaction caused by an excess of catalyst. It indicates that the optimum amount of catalyst is $12 \%$.

\subsubsection{Optimal liquefaction condition of bamboo samples: influence of amount of solvent}

Liquefaction reaction temperatures and amount of catalyst were arranged at $150^{\circ} \mathrm{C}$ and $12 \%$, respectively, and the proportions of bamboo samples to solvent were arranged as $1 / 2,1 / 4$ and $1 / 8$ (the result is shown in Figure 3). It was indicated that the rate of residue decreased as the amount of solvent increased. It is thought that the condensation reaction between the degradation products of the bamboo samples was controlled because the concentration of polyol in the sample was increased by a lot of solvent. When the proportion of bamboo samples to solvent was $1 / 8$, a condensation reaction did not occur at a later reaction stage and the rate of residue was lower than with other proportions. However, in terms of the effective utilization of biomass, it is a problem to contain too low an amount of bamboo samples in the liquefaction product. Therefore, it is indicated that the optimum proportion of bamboo samples to solvent is $1 / 4$.

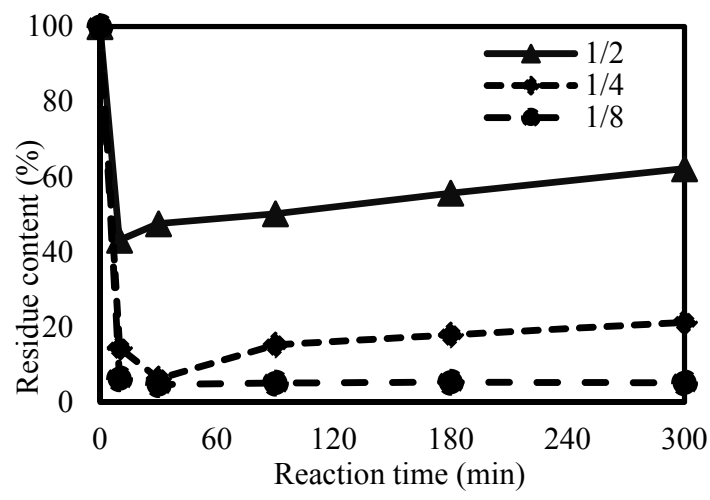

Figure 3: The effect of the solvent amount on residue contents with the different proportions of bamboo samples to solvent arranged as 1/2,1/4 and $1 / 8$.

\subsubsection{The molecular weight distribution of liquefied bamboo samples}

The molecular weight distribution of the liquefied bamboo samples in each reaction time at a reaction temperature of $150^{\circ} \mathrm{C}$, the amount of catalyst of $12 \%$ and $1 / 4$ proportion of bamboo samples to solvent was analyzed using GPC. Condensation reaction occurs in the liquid phase and the solid residue forms from the liquid phase. Therefore, condensation reaction in the liquid phase was investigated by measuring molecular weight distribution of the liquefied bamboo samples (Figure 4). As a result, the peak in reaction time of 10 minutes in the highest molecular weight distribution (a retention time of between 5 and 6 
minutes) became smaller and almost disappeared in a reaction time of 90 minutes. In addition, the big peak at a retention time of 7 minutes became smaller from a reaction time of 30 minutes to 90 minutes and shifted to a retention time of 8 minutes which was a lower molecular weight distribution. In addition, the peak in reaction time of 300 minutes increased in high molecular weight distribution by a condensation reaction in the liquid phase. It is thought that the reason why peak intensity decreased totally while reaction progressed is that existing molecules decreased in the liquid phase by forming solid residues. When the shape of the peaks of reaction time of before 30 minutes and after 90 minutes was compared, the latter accounted for a bigger ratio in high molecular weight distribution. Therefore, it was expected that the average molecular weight was high at a reaction time of after 90 minutes.

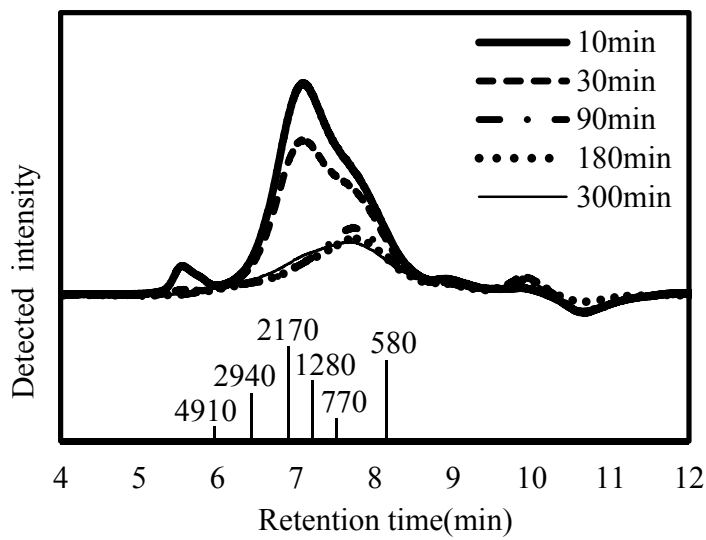

Figure 4: Molecular weight distribution of liquefied bamboo samples determined by GPC analysis after different liquefaction reaction times of 10, 30, 90, 180 and 300 minutes. Here, these values in the bottom of the figure mean the standard molecular weights corresponding to the retention times around 6-8 minutes when liquefied bamboo samples passed through the column during GPC analysis.

\subsection{Analysis and liquefaction of white-rotted woody samples and the measurement of molecular weight distribution of liquefied contents}

\subsubsection{Holocellulose and lignin of white-rotted woody samples}

Holocellulose and lignin of the white-rotted woody samples and non-rotted woody samples were analyzed respectively. Holocellulose and lignin of the white-rotted woody samples were $72.8 \%$ and $27.8 \%$, and those of the non-rotted woody samples were $75.7 \%$ and $22.5 \%$ respectively. The big differences between whiterotted woody samples and non-rotted woody samples were not seen in either holocellulose or lignin. In other words, the big difference did not occur in the weight ratio of woody major components of the white-rotted woody samples and the non-rotted woody samples. Since holocellulose and lignin were degraded by 
white rot fungi, it is suggested that there were no differences in holocellulose and lignin caused by the different degradation mechanism from brown-rot fungi. Regarding the degradation and absorption of the wood by the white-rot fungi, the white-rot fungi degrades cellulose, hemicellulose and lignin at the same time and then the degradation products are absorbed and used immediately [9]. This suggests that non-degraded cellulose, hemicellulose and lignin remained as the same ratio in the rotted woody samples. Thus, this result is conceivable since the weight ratio of components did not even change even when the woody samples were decayed.

\subsubsection{Measurement of crystallinity of white-rotted woody samples}

Crystallinity of holocellulose measured using a XRD is shown in Figure 5. The big differences in the peak between white-rotted woody samples and non-rotted woody samples were seen. From characteristic of measurement using XRD, woody samples are amorphous substance as detection intensity becomes lower. Therefore, the crystal structure of white-rotted woody samples was destroyed by white-rot fungi. Because of the difference between the white-rotted woody samples and the non-rotted woody samples with numerical values, it is the evidence of the white-rotted woody samples which have definitely decayed in the crystallinity. The difference in crystallinity becomes the important factor from this study.

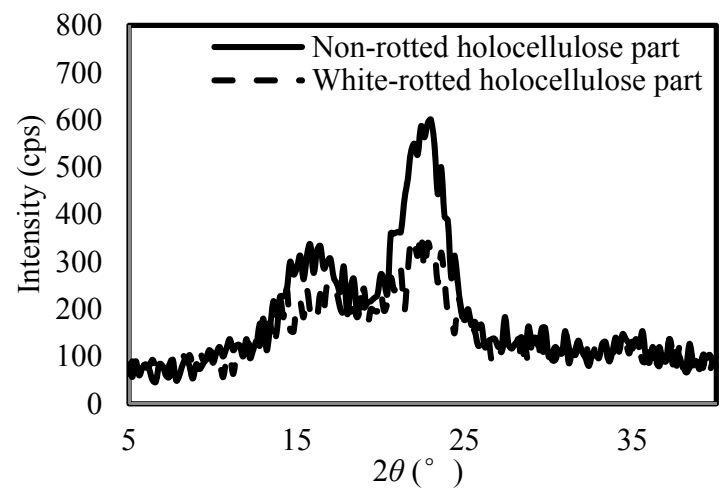

Figure 5: Comparison of crystallinity of white-rotted woody samples and nonrotted woody samples measured using an XRD.

\subsubsection{Rate of residue contents of liquefied white-rotted woody samples}

The rate of residue in the liquefied white-rotted woody samples and the non-rotted woody samples are shown in Figure 6. Reaction condition A was decided at the high reaction temperature of $170^{\circ} \mathrm{C}$, amount of catalyst of $50 \%$ (for the amount of woody samples) and $1 / 2$ proportion of woody samples to solvent. Reaction condition $\mathrm{B}$ was decided at the low reaction temperature of $130^{\circ} \mathrm{C}$, amount of catalyst of $10 \%$ and $1 / 6$ proportion of woody samples to solvent. Condition $\mathrm{A}$ is a relatively intense liquefaction condition while condition $\mathrm{B}$ is a relatively moderate 
liquefaction condition. As a result, the obvious differences between the whiterotted woody samples and the non-rotted woody samples were not seen either under condition $\mathrm{A}$ or under condition $\mathrm{B}$.

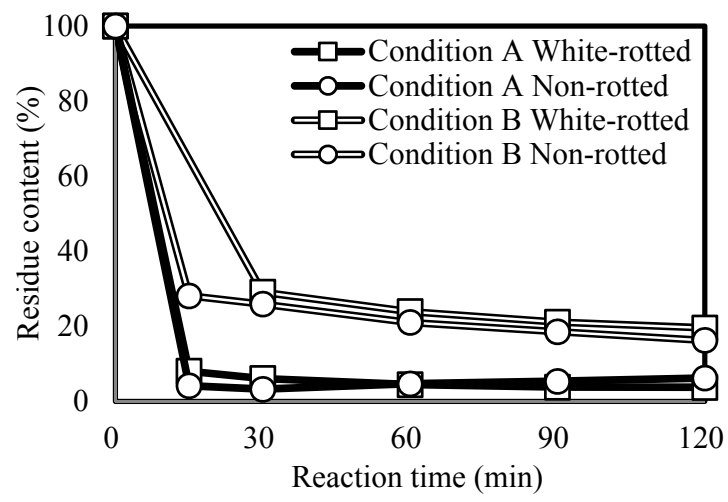

Figure 6: Comparison of residue contents of liquefied white-rotted woody samples and non-rotted woody samples.

Under condition A, the rate of residue of the non-rotted woody samples was seen to increase slightly as reaction progressed after 30 minutes. Because a slight condensation reaction occurred after 120 minutes of reaction time, it is possible to consider that the condensation reaction of these woody samples may be difficult to be produced, like that of cherry. In condition A, because the rate of residue was leveling-off in a low value, it is difficult to judge whether this reaction stopped this value or reacted more. Therefore, condition B was arranged to liquefy only slightly in order to make it easy to judge whether the rate of residue was high or low.

Under condition $\mathrm{B}$, the rate of residue of the non-rotted woody samples in a reaction time of 15 minutes remained at approximately $30 \%$, unlike under condition A. Conversely, the liquefied white-rotted woody samples were not able to be collected in a reaction time of 15 minutes, because all the woody samples were not liquefied immediately when sulfuric acid was added in the reactant and liquefaction reaction was started. The woody samples were liquefied from the place where sulfuric acid was exposed and then a small quantity of the liquefied woody samples was stirred and broke the circumjacent unreacted woody samples little by little. Therefore, a few minutes was needed before all the woody samples in the reactant were liquefied. This phenomenon either occurred slightly under condition A. However, sampling the liquefied woody samples was not affected. It is suggested that the density of the woody samples is related to this. Because rotted wood is degraded and cellulose, hemicellulose and lignin have disappeared by rot fungi, the weight of rotted wood is lighter than that of non-rotted wood despite being the same volume; in the other words, the powder density is small. In this study, because same amount of white-rotted woody samples and non-rotted woody samples was used, the volume of white-rotted woody samples was big. Therefore, 
sulfuric acid did not penetrate immediately all the woody samples and the liquefaction reaction was delayed.

Under condition A, there was no obvious difference between the white-rotted woody samples and the non-rotted woody samples. Under condition B, after the mix of the sample, the tendency of the rate of residue of the white-rotted woody samples was similar to the non-rotted woody samples. Therefore, rotted woody samples can liquefy like non-rotted samples if all of the rotted woody samples react completely with sulfuric acid and solvent.

\subsubsection{Comparing molecular weight distribution of white-rotted woody samples with that of non-rotted woody samples}

A change of the molecular weight distribution was confirmed in the bamboo samples. Moreover, the white-rotted woody samples were compared with the nonrotted woody samples and the molecular weight distribution of the liquefied woody samples was analyzed using GPC analysis. The results are shown in Figures 7 and 8 . Figure 7 is the result of a liquefaction reaction time of 15 minutes, and Figure 8 shows the result of liquefaction reaction time of 30 minutes. Both Figures 7 and 8 are under condition $\mathrm{A}$. The phenol peak appeared at a retention time between 9 and 10 minutes.

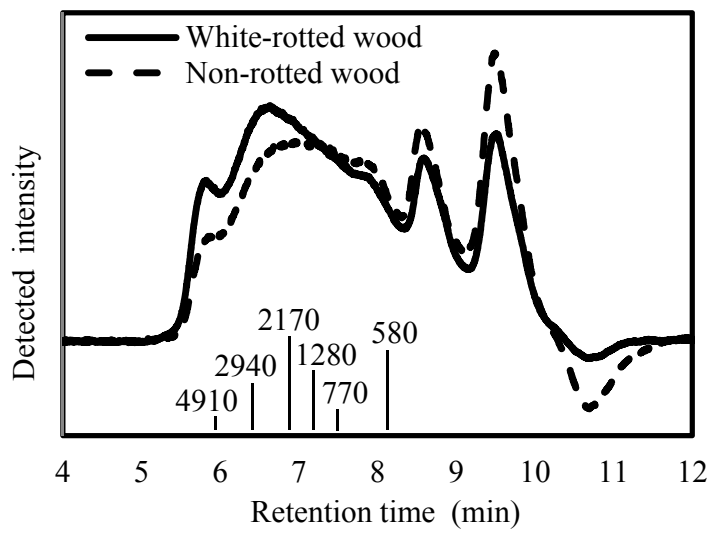

Figure 7: Comparison of molecular weight distribution of liquefied woody samples determined by GPC analysis after 15 minutes' liquefaction reaction. Here, these values at the bottom of the figure mean the standard molecular weights corresponding to the retention times around 6-8 minutes when liquefied bamboo samples passed through the GPC column.

In Figure 7, the peaks at retention time of between 8 and 9 minutes were at the same molecular weight. However, the peak of the white-rotted woody samples at retention time between 5 and 8 minutes appeared in a slightly higher molecular weight distribution. As Figure 8 shows, the peak at retention time of between 5 and 8 minutes did not appear to be the same as those in Figure 7, i.e., both the 
molecular weight distributions of white-rotted woody samples and non-rotted woody samples seemed to be very similar. In addition, the peak of the non-rotted woody samples at a retention time of between 5 and 8 minutes shifted to a higher molecular weight distribution. It is suggested that non-rotted woody samples reached a condensation reaction in liquid phase as mentioned in Section 3.2.3. However, it is also necessary to investigate an additional condensation reaction in order to confirm that it because the phenomenon was only found in Figure 6.

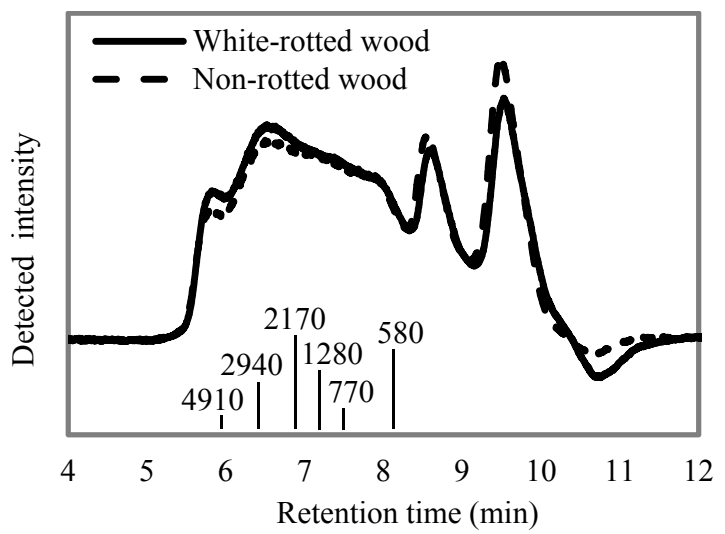

Figure 8: Comparison of molecular weight distribution of liquefied woody samples determined GPC analysis after 30 minutes' liquefaction reaction. Here, these values at the bottom of the figure mean the standard molecular weights corresponding to the retention times of around 6-8 minutes when liquefied bamboo samples passed through the column during the GPC analysis.

\section{Conclusion}

In this paper, the bamboo samples and the white-rotted woody samples were liquefied and the differences in molecular weight distribution were analyzed using GPC analysis. The optimum reaction conditions of bamboo samples, the whiterotted woody and the non-rotted woody samples were investigated.

For the bamboo samples, the optimum liquefaction reaction was found at a reaction temperature of $150^{\circ} \mathrm{C}$, an amount of catalyst of $12 \%$ (for the amount of bamboo samples) and 1/4 proportion of bamboo samples to solvent. As a result of analysis of molecular weight distribution, the rate of peak in the low molecular weight distribution increased during the initial reaction stage of 10-30 minutes because the high molecular weight woody components were well degraded. However, in the later reaction stage of 90-300 minutes, it was determined that the rate of peak in the high molecular weight distribution increased by the condensation reaction in the liquid phase.

In the white-rotted woody samples, no obvious differences appeared when the contents of lignin and holocellulose in the white-rotted woody samples were 
compared with those of the non-rotted woody samples. There were no obvious differences between the white-rotted woody samples and the non-rotted woody samples under both intense and moderate conditions. Although the white-rotted woody samples were added by a catalyst, the reaction didn't start immediately, and it needed the time for all of the woody samples to be liquefied in the moderate liquefaction condition. After all the woody samples reacted completely, similar tendencies were found in both the rate of residue of the white-rotted and the nonrotted woody samples. Furthermore, the differences in the molecular weight distribution between the white-rotted woody samples and the non-rotted woody samples were still not determined using GPC analysis. Consequently, it is found that both bamboo samples and rotted woody samples can be converted into liquefaction products and utilized effectively to substitute the plastic materials made from fossil fuel resources.

\section{Acknowledgement}

Some work in this study is supported by the Special Funds for Basic Research (B) (No. 19404021, FY2007-FY2009, No. 22404022, FY2010-FY2012 and No. 15H05119, FY2015-FY2017) of the Japanese Ministry of Education, Culture, Sports, Science and Technology (MEXT), Japan.

\section{References}

[1] The Japanese Forestry Agency official website (last accessed: 15 April 2015), http://www.rinya.maff.go.jp/ (in Japanese).

[2] Shiraishi, N. et al., Dissolution of etherified wood into polyhydric alcohols or bisphenol A and their application in preparing wooden polymeric materials, Mokuzai gakkaishi, 31, 418-420, 1985.

[3] Y. Yan, et al., Preparation and Characterization of Water-Blown Polyurethane Foams from Liquefied Cornstalk Polyol, Applied Polymer Science, 110, 1099-111, 2008.

[4] S. H. Lee et al., Resol-type phenolic resin from liquefied phenolated wood and its application to phenolic foam, Journal of Applied Polymer Science, 84, 468, 2001.

[5] Gai-Yun Li et al., Preparation and characterization of novolak phenol formaldehyde resin from liquefied brown-rotted wood, Journal of Applied Polymer Science, 125, 3142-3147, 2012.

[6] Q. Wang, et al., Process analysis of the waste bamboo by using polyethylene glycol solvent liquefaction, Sustainable Development and Planning, 9, 647657, 2014.

[7] Nihon Mokuzai Gakkai, 2000, "Mokusitu Kagaku Jikken Maual (wood scientific experiment manual)", published by Bunneido (in Japanese).

[8] Q. Wang, et al., Investigation of condensation reaction during phenol liquefaction of waste woody materials, Sustainable Development and Planning, 9, 658-668, 2014.

[9] Munezoh Takahashi, Fungal Decay Types, Their Significance in Wood Preservation, Wood study, information, 22, 19-36, 1986. 